\title{
Relative ordering in the radial evolution of solar wind turbulence: the S-Theorem approach
}

\author{
G. Consolini ${ }^{1}$ and P. De Michelis ${ }^{2}$ \\ ${ }^{1}$ INAF - Istituto di Fisica dello Spazio Interplanetario, 00133 Roma, Italy \\ ${ }^{2}$ Istituto Nazionale di Geofisica e Vulcanologia, 00143 Roma, Italy
}

Received: 28 February 2011 - Revised: 4 November 2011 - Accepted: 21 November 2011 - Published: 23 December 2011

\begin{abstract}
Over the past few decades scientists have shown growing interest in space plasma complexity and in understanding the turbulence in magnetospheric and interplanetary media. At the beginning of the 1980s, Yu. L. Klimontovich introduced a criterion, named S-Theorem, to evaluate the degree of order in far-from-equilibrium open systems, which applied to hydrodynamic turbulence showed that turbulence flows were more organized than laminar ones. Using the same theorem we have evaluated the variation of the degree of self-organization in both Alfvénic and non-Alfvénic turbulent fluctuations with the radial evolution during a long time interval characterized by a slow solar wind. This analysis seems to show that the radial evolution of turbulent fluctuations is accompanied by a decrease in the degree of order, suggesting that, in the case of slow solar wind, the turbulence decays with radial distance.
\end{abstract}

Keywords. Interplanetary physics (Solar wind plasma) Space plasma physics (Turbulence)

\section{Introduction}

The solar wind results from the expansion of the solar atmosphere, forming a supersonic flow of ionized plasma and magnetic field that permeates the interplanetary medium. It consists mainly of protons and electrons with a small admixture of ionized helium and heavy ions. The solar magnetic field embedded in the plasma is a weak (a few nano Teslas near the Earth) and is carried into space by the solar wind. Although the solar magnetic field is characterized by a complex structure on the Sun within less than 2 solar radii from the photosphere, in the solar wind it exhibits a simple ra-

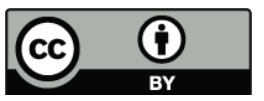

Correspondence to: G. Consolini (giuseppe.consolini@ifsi-roma.inaf.it) dially directed structure lying near the ecliptic plane in an Archimedean spiral pattern.

To treat the solar wind properly many approaches have been tried, each with a different approximation. Indeed, it is possible to study the solar wind using a fluid approximation or a kinetic treatment; however, in both cases difficulties exist (Russel, 2001). The result is that our understanding of the solar wind is nowadays founded on long term observations rather than on solid theoretical foundations.

The solar wind was measured for the first time in the 1960s with the advent of spacecrafts. After the first measurements, it was clear that it was pervaded by fluctuations on a very wide range of scales. The subsequent studies of these fluctuations revealed a complex interaction among waves, turbulence and structures (see e.g. Bruno and Carbone, 2005). Nowadays, it is known that the solar wind is characterized by the presence of fluctuations in the magnetic and velocity fields that are often so highly correlated to appear as nearly perfect Alfvén waves. The solar wind shows an active turbulent cascade, which transfers energy from large scales to small scales and heats the plasma (Verma et al., 1995; Marino et al., 2008). Finally, it exhibits a turbulence which is anisotropic with respect to the magnetic field and it is also characterized by intermittency similar to that found in neutral fluids.

Focusing on the turbulent nature of the solar wind, the power spectrum of solar wind fluctuations provides one of the main evidences for the existence of an active turbulent cascade (Bruno and Carbone, 2005). Indeed, the power spectrum of fluctuations of the magnetic field components, evaluated in the case of high speed solar wind, reveals broadband fluctuations over all measured scales. These fluctuations are characterized by a power law spectrum, which depends on the frequency range: at low frequencies the power law exhibits a spectral index near 1 while at higher frequencies the spectral index is near $5 / 3$. The presence of a broad range of scales with an $f^{-5 / 3}$ spectrum may be associated with a

Published by Copernicus Publications on behalf of the European Geosciences Union. 
turbulent cascade, being $5 / 3$ the familiar value of the spectral index in hydrodynamic turbulence. This turbulence follows from processes occurring in the solar wind and does not simply reflect the nature of the solar wind emerging from the solar corona. Indeed, according to Bavassano et al. (1982a) the power spectrum of fluctuations changes its shape with distance from the Sun, as the breakpoint between the two regimes $f^{-1}$ and $f^{-5 / 3}$ shifts to lower frequencies (i.e. larger scales) with the increase of solar distance. Thus, the fluctuation properties evolve with both time and radial distance. Together with changes in the power spectrum with distance, there is a decrease in the Alfvénicity character of the fluctuations (Roberts et al., 1987), which seems to be closely related to the turbulent evolution. These results characterize mainly the fast solar wind. Conversely, the slow solar wind spectra are characterized by a spectral index near $5 / 3$ over a wide range of scales. The broadband $f^{-5 / 3}$ spectrum associated with a generally low Alfvénicity (Tu et al., 1989) suggests that slow solar wind fluctuations represent fully developed turbulence. For this reason, the solar wind evolution cannot be reduced to a simple solar wind expansion with the heliocentric distance, but it involves nonlinear interactions among propagating Alfvénic modes (Tu et al., 1984). It has been shown that intermittency increases with radial distance, probably due to a change in the anisotropy degree of the magnetic fluctuations. However, contradictory results have been found as regards the evolution of the anisotropy degree with radial distance. For instance, Bavassano et al. (1982b) showed that in high-speed streams the orthogonal component of the magnetic field fluctuations became more dominant with the increase of the distance, while Horbury et al. (1995) claimed that turbulence was more isotropic at larger heliospheric distances in the case of polar solar wind.

Besides turbulence an important aspect characterizing the solar wind is the presence of coherent advected structures, which can be imagined like flux tubes (Ness et al., 1966; McCracken and Ness, 1966; Mariani et al., 1973; Tu and Marsch, 1990, 1993; Bruno et al., 2001; Borovsky, 2008). The presence of such non-Alfvénic coherent structures, whose origin is not completely understood, becomes more relevant with radial distance (Bruno et al., 2006).

Recently, Consolini (2010) examined the radial evolution of magnetic field intensity fluctuations during a long time interval characterized by a slow solar wind. This analysis has been based on the S-Theorem by Klimontovich (1983) in order to examine the changes of the degree of order of the magnetic field intensity fluctuations at two heliospheric distances. The preliminary results have pointed toward an increase in the degree of order with radial distance, which suggests the formation of coherent structures during the radial evolution of magnetic field intensity fluctuations.

Here, analyzing the same long time interval of slow solar wind investigated by Consolini (2010), we carry out an indepth analysis of the relative degree of order of the turbulent fluctuation field. In detail, we investigate the changes in the probability distribution functions (PDFs) of the total specific energy of turbulent fluctuations on the time scale of $1 \mathrm{~h}$ with radial distance, demonstrating that the radial evolution of turbulent fluctuations is associated with a chaotization process.

The paper is organized as follows: in Sect. 2, we briefly describe the Klimontovich S-Theorem; in Sect. 3 we apply such a theorem to our data and in Sect. 4 we discuss and summarize our results.

\section{Klimontovich' S-Theorem: a brief introduction}

In thermodynamics and statistical mechanics the state of maximum disorder corresponds to the equilibrium state for a given condition of the external parameters, as clearly stated by the principle of maximum entropy. Thus, any deviation from the equilibrium configuration is associated with a reduction of entropy. This happens, for instance, in nonequilibrium stationary states, where the degree of order can increase due to the emergence of a spatial-temporal coherence. Indeed, in many nonequilibrium systems we assist to the emergence of correlations and self-organization that imply a reduction of the physical chaos (uncorrelated microscopic motion). In this framework, a crucial importance assumes the knowledge of a method able to quantify the relative degree of order in different open nonequilibrium states of the same system.

Over the last two decades the concept of dynamic chaos has gained a particular relevance in the framework of complex motions in relatively simple systems. Dynamic chaos, which is consequence of dynamic instabilities, has not to be confused with physical chaos, which is related to the absence of coherence in the motion of particles in a state of thermodynamic equilibrium according to the original definition by Boltzmann. Consequently, it would be interesting to have a criterium to estimate quantitatively if the dynamic chaos, emerging from dynamic instabilities, could or not lead to more ordered configurations.

In 1983 Klimontovich (Klimontovich, 1983, 1995) proposed an entropy-based approach to measure the relative degree of order between two different states in an open nonequilibrium system. This approach, formulated as a theorem, named the Self-organization Theorem (S-Theorem), attempts to solve one of the main tasks of the statistical theory of open systems, establishing a criterium capable of measuring the degree of self-organization of an open system in a nonequilibrium configuration. The aim of the S-Theorem is to distinguish between degradation and self-organization in different nonequilibrium configurations of open systems, thus providing a quantitative measure of order with respect to physical chaos (equilibrium).

The starting point of the $S$-Theorem is the Boltzmann's $H$-theorem, according to which the entropy variation $\Lambda_{S}$ 
between an equilibrium state and a nonequilibrium one in a system with a fixed value of mean energy is

$\Lambda_{\mathrm{S}}=S_{0}-S(t)=\kappa_{\mathrm{B}} \int f(X, t) \ln \frac{f(X, t)}{f_{0}(X)} d X \geq 0$

under the condition,

$\frac{d \Lambda_{\mathrm{S}}}{d t}=\frac{d}{d t}\left(S_{0}-S(t)\right) \leq 0$,

being $\kappa_{\mathrm{B}}$ is the Boltzmann's constant, $S_{0}\left(f_{0}(X)\right)$ and $S(t)$ $(f(X, t))$ the entropies (distribution functions) of the equilibrium and nonequilibrium states, respectively. This theorem states that a system with fixed mean energy evolves towards an increase of entropy, which reaches its maximum at the equilibrium state. Based on the condition of fixed mean energy the above relationship implies that the entropy difference $\Lambda_{\mathrm{S}}(t)$ acts as a Lyapunov functional for the evolution of a system.

However, the entropy reduction $\Lambda_{\mathrm{S}}$ is inadequate to estimate the relative degree of order in systems whose states depend on a control parameter that yields different mean energy values. This is particularly true in the case of open systems where the mean energy is generally not constant during the evolution. For these types of systems an appropriate Lyapunov functional for the evolution is impossible to define correctly in terms of entropy difference. In other words, it is not possible to evaluate the relative degree of order between two open systems in different nonequilibrium states using the previous relationship. Klimontovich's S-Theorem finds a solution to this problem renormalizing the distribution of the reference state and making use of escort distributions. The $\mathrm{S}$-Theorem provides a measure of the degree of order relative to a reference state for open systems, thereby supplying the correct ordering of entropy values with respect to their distance from the equilibrium state.

Given two different nonequilibrium states, $\mathcal{A}\left(a_{0}\right)$ and $\mathcal{A}\left(a_{0}+\delta a\right)$, of the same open system, which correspond to two different values $\left(a_{0}\right.$ and $\left.a_{0}+\delta a\right)$ of a controlling parameter, we indicate with $f\left(X ; a_{0}\right)$ and $f\left(X ; a_{0}+\delta a\right)$ the correspondent time independent distribution functions for the observable $X$ based on the hypothesis of stationary external conditions (i.e. a time-scale separation between fast processes and the slow background evolution). To take into account the possible change of the mean energy it is necessary to renormalize the distribution function associated with one of the two states, taken as the reference state, before evaluating the entropy reduction (Klimontovich, 1995).

Thus, we assume as reference state (i.e. as the most chaotic state) the one with $a=a_{0}$ and identify with $f_{0} \equiv f\left(X ; a_{0}\right)$ its distribution function, and with $f_{1} \equiv\left(X ; a_{0}+\delta a\right)$ the distribution function of the other state. Following Klimontovich's procedure, we introduce an effective Hamiltonian or energy $H_{\text {eff }}\left(X ; a_{0}\right)$ as

$H_{\text {eff }}\left(X ; a_{0}\right)=-\ln f_{0}\left(X ; a_{0}\right)$.
To ensure that the mean effective energy of the reference state is equal to that associated with the other state, we have to renormalize the reference state distribution function as follows,

$\tilde{f}_{0}\left(X ; a_{0}, \delta a\right)=\exp \left[\frac{F_{\text {eff }}-H_{\text {eff }}\left(X ; a_{0}\right)}{T_{\text {eff }}(\delta a)}\right]$,

where $F_{\text {eff }}$ is the effective free energy that can be expressed in terms of the effective temperature $T_{\mathrm{eff}}$ on the basis of the normalization condition,

$\int \tilde{f}_{0}\left(X ; a_{0}, \delta a\right) d X=1$.

It follows

$F_{\text {eff }}=-T_{\text {eff }} \ln \int \exp \left(-\frac{H_{\text {eff }}\left(X ; a_{0}\right)}{T_{\text {eff }}}\right) d X$.

We remark that the effective Hamilton function $H_{\mathrm{eff}}$ is not representative of the conventional concept of energy.

Based on the previous mathematical assumptions, the only free parameter is the effective temperature $T_{\text {eff }}(\delta a)$, which can be determined by imposing that the two distribution functions $\tilde{f}_{0}$ and $f_{1}$ have the same mean effective energy,

$\int H_{\mathrm{eff}} \tilde{f}_{0}\left(X ; a_{0}, \delta a\right) d X=\int H_{\mathrm{eff}} f_{1}\left(X ; a_{0}+\delta a\right) d X$.

Consequently, we have

$\left.T_{\mathrm{eff}}(\delta a)\right|_{\delta a=0}=1$,

where the condition $\delta a=0$ refers to the reference state.

Once we have renormalized the reference state distribution function according to the condition of constant effective mean energy, we obtain two distribution functions $\tilde{f}_{0}$ and $f_{1}$, with the same mean energy. The entropy difference between the two states can be evaluated using Eq. (1), i.e.

$\delta S=\tilde{S}_{0}-S=\int f_{1} \ln \frac{f_{1}}{\tilde{f}_{0}} d X$,

where $\delta S \geq 0$ if we made the right choice of the reference state of physical chaos.

The solution of Eq. (7) provides a quantitative measure of the relative order between two selected states. Indeed, if the condition of Eq. (7) is satisfied for $T_{\text {eff }}(\delta a)>1$, we will have to raise the effective temperature and add heat to the reference state " 0 " to modify it into the more organized state " 1 ". Thus, the state " 0 " is a state with more physical chaos than the state " 1 ". To clarify the meaning of the effective temperature $T_{\text {eff }}$ as a measure of the degree of order we invite the reader to consult the Appendix A where we make all the above calculations in the case of a simple system. We note that the concept of state of maximum physical chaos has to be associate with the equilibrium state, where according to Boltzmann the particle motion is chaotic and generally uncorrelated. In other words, the term "physical chaos" is 

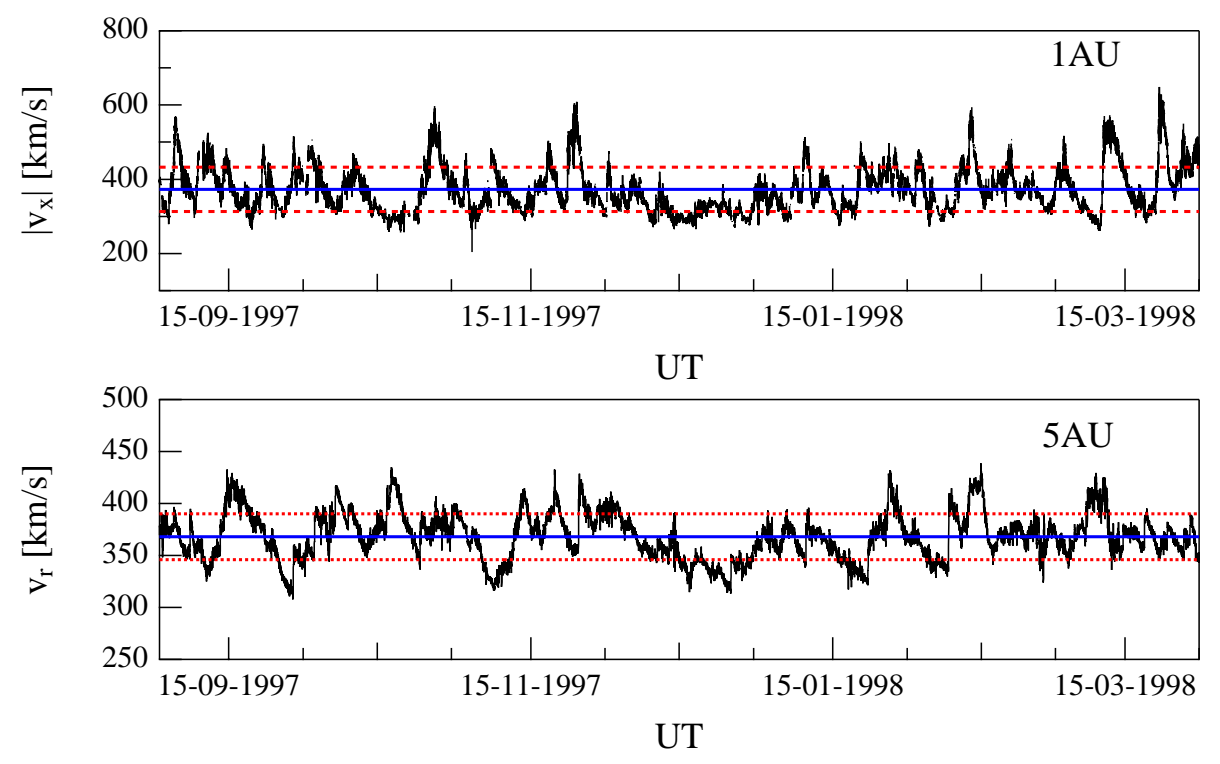

Fig. 1. The X-component of the solar wind velocity at $1 \mathrm{AU}$ (upper panel) and the radial component of the solar wind velocity at 5 AU (lower panel) for the period from 1 September 1997 to 31 March 1998. Horizontal solid blue lines indicate mean values (blue line); dashed lines limit \pm 1 standard deviation intervals.

directly connected with uncorrelated stochastic motions and not with the sensitivity to initial conditions.

We emphasize that in nonequilibrium systems the emergence of complexity and dynamical complexity is identified with the tendency to manifest spatio-temporal coherent structures and features. These generally result from the competition of different basic spatial patterns (Badii and Politi, 1997), and require the intertwining of order and disorder (Nicolis and Nicolis, 2007). For instance, this happens in the case of turbulence, where the turbulent eddies are coherent structures. On the other hand, the existence of coherent structures in a system is the counterpart of a self-organization process, which increases the coherence and the correlation in the system and reduces the degree of disorder or physical chaos, i.e. uncorrelated motions. (Klimontovich, 1991, 1996). Thus, it is reasonable to observe a significant reduction of the degree of disorder in a complex system, in agreement with the S-theorem.

\section{Data and results}

To study the relative order and the occurrence of selforganization in the solar wind turbulence radial evolution we consider the hourly variances of the interplanetary magnetic and velocity fields measured in situ at $1 \mathrm{AU}$ and at $\sim 5 \mathrm{AU}$ in the time interval September 1997-March 1998. Data at $1 \mathrm{AU}$ come from the OMNI database (NSSDC-USA), while data at $\sim 5 \mathrm{AU}$ come from the Ulysses measurements, available at NASA-CDAweb. Typical data time resolution is $1 \mathrm{~min}$ apart from Ulysses velocity which is at about $4-8 \mathrm{~min}$.
The chosen period is a long-lasting time interval of quiet solar wind conditions, already investigated and discussed in previous works (Consolini et al., 2008; Consolini, 2010). During this period the solar wind velocity is slow and more or less constant with small gradients (see Fig. 1). For this reason, as a first approximation, we can assume that the solar wind conditions are quasi-stationary. The average velocity is $\left\langle v_{\mathrm{x}}\right\rangle=[370 \pm 60] \mathrm{km} \mathrm{s}^{-1}$ at $1 \mathrm{AU}$ and $\left\langle v_{\mathrm{R}}\right\rangle=[370 \pm$ 20] $\mathrm{km} \mathrm{s}^{-1}$ at $5 \mathrm{AU}$.

Due to the absence of a long-lasting alignment between the two observational points at $1 \mathrm{AU}$ and $5 \mathrm{AU}$, we are not observing the same solar wind, but the choice of such a long period will guarantee a statistical validity to our analysis. To take account the approx. 20 days solar wind traveling time from $1 \mathrm{AU}$ to $5 \mathrm{AU}$, we cut-off the last 20 days from the $1 \mathrm{AU}$ data and the first 20 days from the $5 \mathrm{AU}$ data.

To analyze the evolution of the turbulent solar wind features with the radial distance, we evaluate the $1 \mathrm{~h}$ variances of the plasma velocity and solar wind magnetic field expressed in Alfvén units $(\boldsymbol{B} \rightarrow \boldsymbol{B} / \sqrt{4 \pi \rho}$ with $\rho$ plasma density), i.e. $\left\langle\mathbf{v}^{2}\right\rangle_{1 \mathrm{~h}}$ and $\left\langle\mathbf{b}^{2}\right\rangle_{1 \mathrm{~h}}$, where $\mathbf{v}=\boldsymbol{V}-\langle\boldsymbol{V}\rangle_{1 \mathrm{~h}}$ and $\mathbf{b}=\boldsymbol{B}-\langle\boldsymbol{B}\rangle_{1 \mathrm{~h}}$.

We identify the total specific energy $E_{\mathrm{t}}$ of the fluctuation field with the dynamical variable capable of representing the turbulence level

$E_{\mathrm{t}}=E_{\mathrm{v}}+E_{\mathrm{b}}$.

This dynamical variable takes into account of the kinetic $\left(E_{\mathrm{V}}\right)$ and magnetic $\left(E_{\mathrm{b}}\right)$ specific energies, defined as

$E_{\mathrm{v}}=\frac{1}{2}\left\langle\mathbf{v}^{2}\right\rangle_{1 \mathrm{~h}}$, 

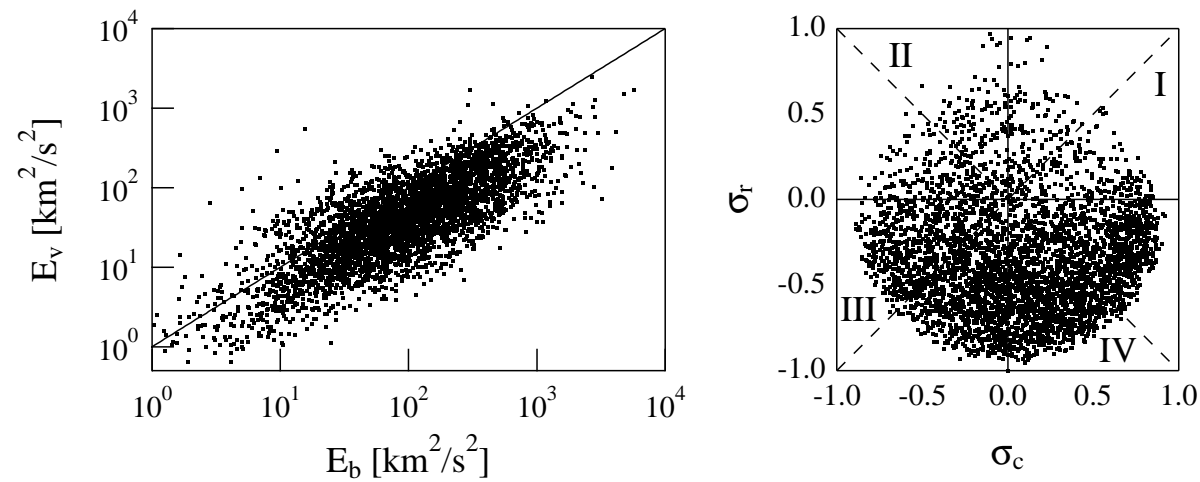

Fig. 2. The kinetic specific energy $E_{\mathrm{v}}$ plotted versus the magnetic specific energy $E_{\mathrm{b}}$ (left panel) and the scatter plot of the normalized residual energy $\sigma_{\mathrm{r}}$ versus the normalized cross-helicity $\sigma_{\mathrm{c}}$ (right panel) for the sample at $1 \mathrm{AU}$. The solid line in the $E_{\mathrm{V}}-E_{\mathrm{b}}$ plot refers to the condition of complete equipartition.
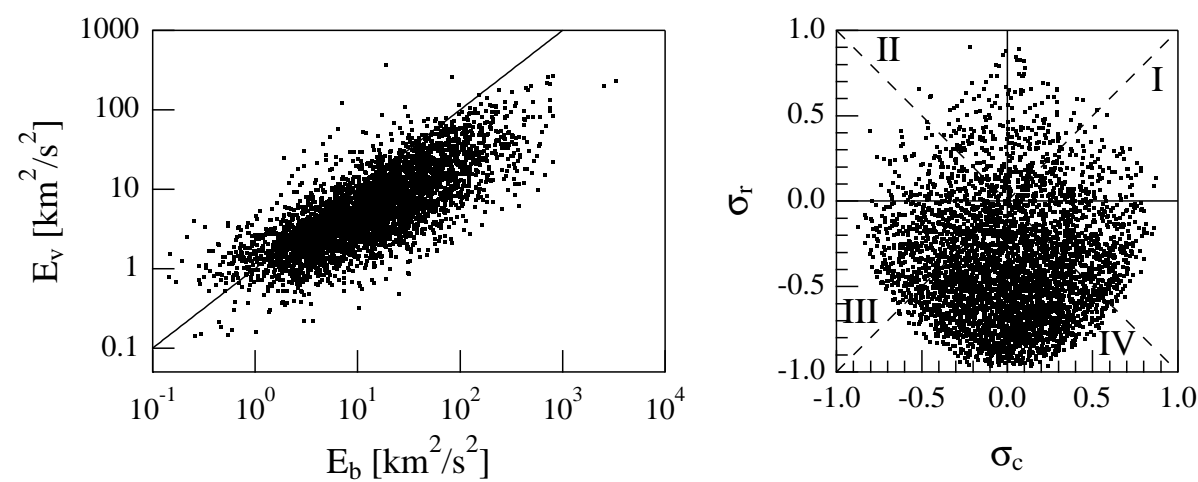

Fig. 3. The same of Fig. 2 for the sample at 5 AU.

$E_{\mathrm{b}}=\frac{1}{2}\left\langle\mathbf{b}^{2}\right\rangle_{1 \mathrm{~h}}$.

Furthermore to recognize the Alfvénic part in the solar wind fluctuations we compute the normalized cross-helicity $\sigma_{\mathrm{c}}$ and the normalized residual energy $\sigma_{\mathrm{r}}$,

$\sigma_{\mathrm{c}}=\frac{2\langle\mathbf{v} \cdot \mathbf{b}\rangle_{1 \mathrm{~h}}}{\left\langle\mathbf{v}^{2}\right\rangle_{1 \mathrm{~h}}+\left\langle\mathbf{b}^{2}\right\rangle_{1 \mathrm{~h}}}$,

$\sigma_{\mathrm{r}}=\frac{E_{\mathrm{v}}-E_{\mathrm{b}}}{E_{\mathrm{t}}}$.

Here, the normalized cross-helicity $\sigma_{\mathrm{c}}$, which can vary from -1 to +1 , quantifies the degree of correlation between the velocity and magnetic field fluctuations, expected to be maximum for Alfvénic fluctuations, while the normalized residual energy is a measure of the energy equipartition between the kinetic and magnetic fluctuations (Bavassano et al., 2000).

Figures 2 and 3 exhibit the kinetic specific energy $E_{\mathrm{v}}$ as a function of the magnetic specific energy $E_{\mathrm{b}}$ and the normalized residual energy $\sigma_{\mathrm{r}}$ versus the normalized cross-helicity $\sigma_{\mathrm{c}}$ for both the data samples at $1 \mathrm{AU}$ and $5 \mathrm{AU}$. The energy equipartition is missed both at $1 \mathrm{AU}$ and $5 \mathrm{AU}$. The $E_{\mathrm{b}}$ values are generally higher than the corresponding $E_{\mathrm{V}}$ ones. This result suggests that a significant part of the to- tal energy of fluctuations comes from magnetic component when a time scale of $1 \mathrm{~h}$ is considered. This is more evident at $5 \mathrm{AU}$ than at $1 \mathrm{AU}$. Indeed, the density of dots is higher at $5 \mathrm{AU}$ than at $1 \mathrm{AU}$ in the IV quadrant of the $\sigma_{\mathrm{r}}-\sigma_{\mathrm{c}}$ plane, and the mean value of the ratio between $E_{\mathrm{v}}$ and $E_{\mathrm{b}}$ is higher at $1 \mathrm{AU}\left(\left\langle E_{\mathrm{v}} / E_{\mathrm{b}}\right\rangle_{1 \mathrm{AU}}=[0.70 \pm 0.02]\right)$ than at $5 \mathrm{AU}$ $\left(\left\langle E_{\mathrm{v}} / E_{\mathrm{b}}\right\rangle_{5 \mathrm{AU}}=[0.64 \pm 0.01]\right)$. Furthermore, according to Fig. 3 the results relative to $\sigma_{\mathrm{c}}$ and $\sigma_{\mathrm{r}}$ well agree with previous findings by Consolini et al. (2008), relative to a predominance of magnetic fluctuations at $5 \mathrm{AU}$.

Now, we evaluate the probability distribution functions (PDFs) of the total specific energy $\left(E_{\mathrm{t}}\right)$ at the two different solar distances and, as special cases, the PDFs relative to Alfvénic and non-Alfvénic fluctuations. The selection of Alfvénic/non-Alfvénic fluctuations is based on $\sigma_{\mathrm{r}}$ and $\sigma_{\mathrm{c}}$ values. For example, only data with $0.2 \leq\left|\sigma_{\mathrm{c}}\right| \leq 1$ and $\left|\sigma_{\mathrm{r}}\right| \leq\left|\sigma_{\mathrm{c}}\right|$ are considered for Alfvénic fluctuations, where the choice $\left|\sigma_{\mathrm{c}}\right|^{\mathrm{min}}=0.2$, as the inferior limit of a significative normalized cross-correlation value, is based on $5 \%$ nullhypothesys threshold for uncorrelated 3-D-vectors. In contrast, those intervals where $\left|\sigma_{\mathrm{c}}\right| \leq\left|\sigma_{\mathrm{r}}\right|$ and $0.2 \leq\left|\sigma_{\mathrm{r}}\right| \leq 1$ are the values used for the selection of non-Alvénic fluctuations. 


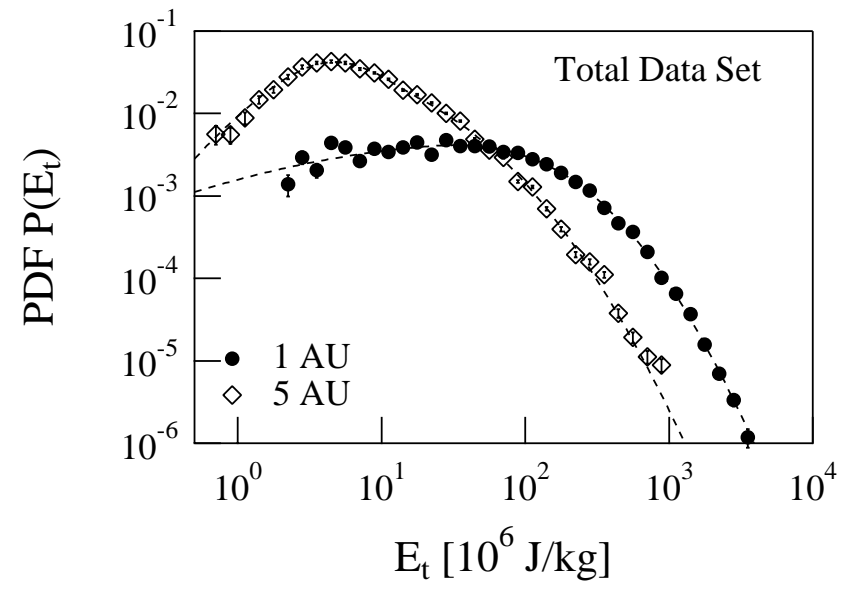

Fig. 4. The probability distribution functions $P\left(E_{\mathrm{t}}\right)$ of the total specific energy $E_{\mathrm{t}}$ for the two distances. Dashed lines are empirical model fits using expression of Eq. (15).

In Figs. 4 and 5 we report the PDFs $P\left(E_{\mathrm{t}}\right)$ of the total specific energy $E_{\mathrm{t}}$ associated with the 3 different data sets: whole data set, Alfvénic and non-Alfvénic subsets. These PDFs are evaluated at the two different radial distances of $1 \mathrm{AU}$ and $5 \mathrm{AU}$. The differences among the PDFs at the same solar distance are not relevant. In contrast, a large effect on the distribution shapes is observed with the change of the radial distance. The shape of the PDFs at 5AU appears less flatten than that at $1 \mathrm{AU}$, suggesting that the radial propagation of solar wind implies an evolution of the physical properties apart from those related to expansion.

In order to estimate the relative degree of order for the two radial distances through the S-theorem, we fit the PDFs using an empirical model able to reproduce accurately the overall shape of the PDFs. In this way, we reduce some computational problems due to the discrete nature of PDFs. We choose the following empirical shape for the PDFs,

$P(x)=A_{1} x^{\alpha} e^{-\left(x / x_{0}\right)^{\beta}}+A_{2} e^{-\left((\ln x-a)^{2} / \sigma\right)}$.

This choice, which is done for computational reasons without any physical consideration, provides PDFs that agree with the actual PDFs. Furthermore, the obtained results are not influenced by the PDFs empirical shape, and agree with those obtained analysing the actual discrete PDFs computed by histogram method.

The condition of constant mean effective energy (i.e. Eq. 7) can be expressed using Eqs. (4) and (6) in terms of $f_{0}, f_{1}$ and $T_{\text {eff }}$,

$\int \frac{f_{0}^{\frac{1}{T_{\text {eff }}}}}{\int f_{0}^{\frac{1}{T_{\text {eff }}}} d X^{\prime}} \ln f_{0} d X=\int f_{1} \ln f_{0} d X$.

Equation (16) can be solved numerically for the effective temperature $T_{\text {eff }}$ once the reference distribution $f_{0}$ is chosen.
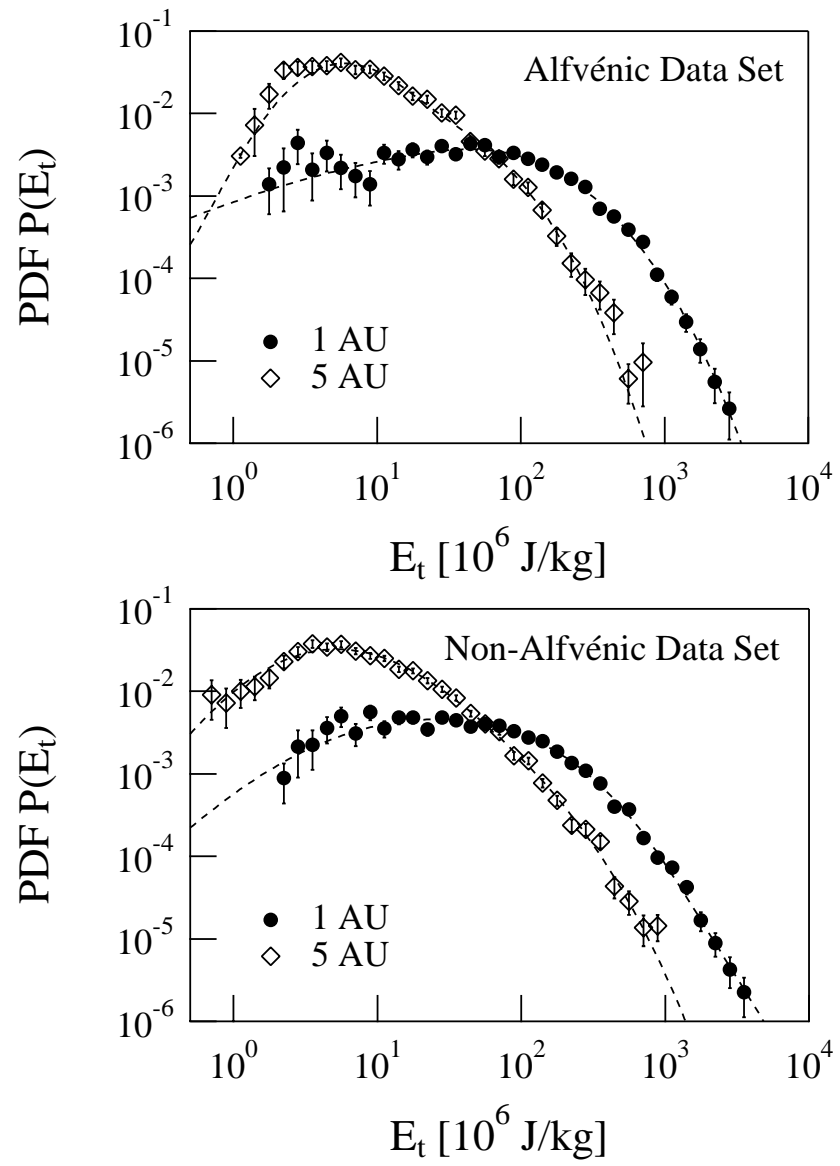

Fig. 5. The probability distribution functions $P\left(E_{\mathrm{t}}\right)$ of the total specific energy $E_{\mathrm{t}}$, relative to Alfvénic fluctuations (upper panel) and non-Alfvénic fluctuations (lower panel), for the two distances. Dashed lines are empirical model fits using Eq. (15).

An example of numerical solution of Eq. (16) is reported in Fig. 6. In this case, we consider the total data set and select as PDF of the reference state that relative to $1 \mathrm{AU}$. The quantities $I_{1}$ and $I_{2}(T)$ are

$$
\begin{aligned}
I_{1} & \equiv \int f_{1} \ln f_{0} d X, \\
I_{2}(T) & \equiv \int \frac{f_{0}^{\frac{1}{T}}}{\int f_{0}^{\frac{1}{T}} d X^{\prime}} \ln f_{0} d X,
\end{aligned}
$$

and permit us to find the effective temperature $T_{\text {eff }}$ by imposing $I_{2}\left(T_{\text {eff }}\right)=I_{1}$. The numerical value of the effective temperature obtained from this case is less than $1\left(T_{\text {eff }}=0.36\right)$. Consequently, our choice to consider the PDF at $1 \mathrm{AU}$ as the reference one is not right. The PDF at $1 \mathrm{AU}$ is not associated with the situation of maximum physical chaos as it has been supposed.

The effective temperatures, obtained from the numerical solution of Eq. (16) are reported in Table 1, assuming as reference both the $1 \mathrm{AU}-\mathrm{PDF}$ and the 5 AU-PDF. Effective 


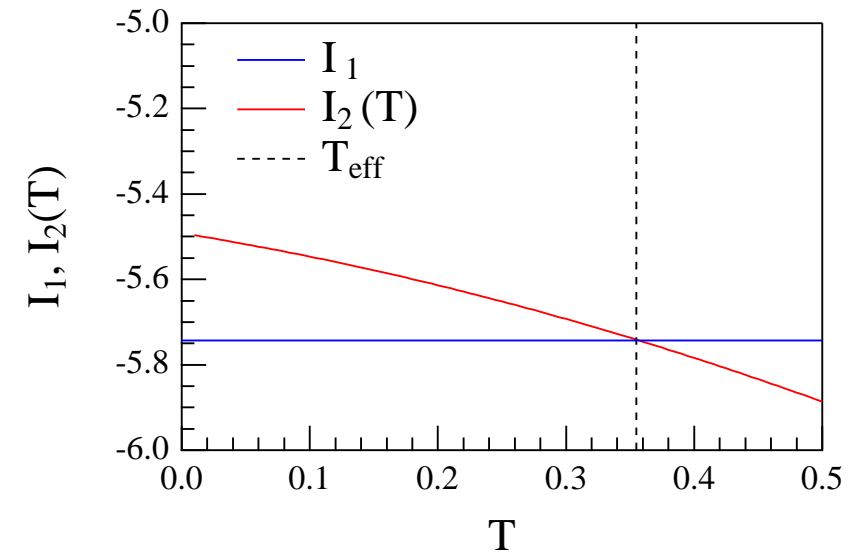

Fig. 6. Plot of $I_{1}$ and $I_{2}(T)$ in the case of the total data set and choosing $1 \mathrm{AU}$ PDF as the reference one $\left(f_{0}\right)$. The intersection between $I_{1}$ and $I_{2}(T)$ provides the value of the effective temperature $T_{\text {eff }}$ that solves Eq. (16) - vertical dashed line.

temperatures greater than 1 are obtained only assuming for $f_{0}$ the PDFs at $5 \mathrm{AU}$.

According to Klimontovich's S-Theorem, the process of self-organization evolves towards the state in which $T_{\text {eff }}>1$. Consequently our results (see Table 1) suggest that the solar wind turbulent fluctuations correspond to a situation of less order (i.e. of higher physical chaos) at $5 \mathrm{AU}$ than at $1 \mathrm{AU}$. In other words, the evolution of the solar wind turbulence with radial distance can be thought in terms of a chaotization process.

Using Eq. (9) we estimate the entropy reduction $\delta S=$ $\tilde{S}_{0}-S$, obtaining $\delta S=0.129, \delta S=0.062$ and $\delta S=0.250$ for the total, Afvénic and non-Alfvénic dataset, respectively. The entropy reduction is positive in all cases, although the largest variation is associated with the non-Alfvénic dataset. This result clearly indicates how the evolution of the solar wind turbulent fluctuations is accompanied by an entropization process.

\section{Conclusions}

The application of the S-Theorem to the radial evolution of the slow solar wind turbulence indicates that the state of the fluctuations of the solar wind at $1 \mathrm{AU}$ is characterized by an higher degree of order than the state of the fluctuations at $5 \mathrm{AU}$. In other words, the slow solar wind fluctuation field exhibits a higher degree of self-organization (coherence) and complexity at $1 \mathrm{AU}$ than at $5 \mathrm{AU}$. Thus, the radial evolution of the solar wind is accompanied by an entropization process, i.e. an increase in physical chaos and an energy degradation with radial distance in the fluctuation field. We emphasize that our analysis provides a quantitative evaluation of the coherence loss of the solar wind fluctuation field with radial distance by means of the entropy reduction $\delta S$. The
Table 1. The effective temperature $T_{\text {eff }}$ as obtained by numerically solving Eq. (16)

\begin{tabular}{llll}
\hline$f_{0} \rightarrow f_{1}$ & Total & Alfvénic & non-Alfvénic \\
\hline $1 \mathrm{AU} \rightarrow 5 \mathrm{AU}$ & $T_{\text {eff }}=0.36$ & $T_{\text {eff }}=0.27$ & $T_{\text {eff }}=0.45$ \\
$5 \mathrm{AU} \rightarrow 1 \mathrm{AU}$ & $T_{\text {eff }}=2.06$ & $T_{\text {eff }}=2.94$ & $T_{\text {eff }}=2.05$ \\
\hline
\end{tabular}

observed energy degradation, which is the signature of a loss of coherence in the fluctuations of the magnetic and velocity field, could be the counterpart of a conversion of energy into heat, which shows up as an uncorrelated and less coherent fluctuation field.

Recently, some works have appeared on the relation between the dependence of the solar wind temperature on the radial distance, and the possible role that turbulent energy transfer rate could play in solar-wind heating (Marino et al., 2008). The first models of solar wind expansion in the heliosphere were based on the hypothesis of a simple adiabatic expansion. According to these models the proton temperature was expected to decrease with the heliocentric distance $r$ as $T(r) \sim r^{-4 / 3}$. Nevertheless, the first spacecraft observations revealed a decrease in the solar wind proton temperature slower than expected (Schwenn, 1983; Freeman et al., 1992; Goldstein et al., 1996), i.e. $T \sim r^{-\gamma}$ with $\gamma \in(0.7,1.0)$. The highly non-adiabatic temperature profile suggested that some heating mechanism is at play to supply energy to the solar wind (Verma et al., 1995). In this framework, a recent study by Marino et al. (2008) has provided evidence of the role of the MHD turbulent cascade in solar wind heating, suggesting that the MHD turbulent energy cascade may contribute from $8 \%$ up to $50 \%$ to in situ solar wind heating.

Our finding of a degradation of the fluctuation field with radial distance could be, to some extent, related to the results of Marino et al. (2008). Indeed, the reduction of the self-organization degree in favor of a state characterized by a higher physical chaos would imply a transformation of the energy related with correlated motions into heat. However, in our analysis the most relevant loss of coherence with radial distance is found in the case of non-Alfvénic fluctuations $(\delta S=0.250)$. Thus, if the suggestion of an active role of MHD turbulence in solar wind heating is accepted, not only the MHD turbulence but also other types of energy degradation mechanisms will contribute to solar wind heating in a consistent manner. Clearly, at the present stage, this hypothesis is only speculative. It is, indeed, not possible from our analysis to infer the physical mechanisms which are capable of converting into heat the loss of coherence and the energy degradation in the fluctuation field. The possible link between particle heating and chaotization of the fluctuation field demands a more accurate theoretical investigation that is beyond of the aim of this work. 
In connection with the evolution of the turbulence anisotropy character, the observed loss of coherence could suggest that, in agreement with the results of Horbury et al. (1995) for the polar wind, the radial evolution is accompanied by an increase in isotropy at larger heliospheric distances also in the case of slow equatorial solar wind. Moreover, the overall decrease in the degree of order with radial distance suggests that we could be in the presence of a process of decaying turbulence in the case of slow solar wind. This hypothesis is supported by the following arguments.

The S-Theorem application to hydrodynamic turbulent flows (Prigogine and Stengers, 1984; Ebeling and Klimontovich, 1984; Klimontovich, 1984; Ebeling, 1989) demonstrates that, in contrast to the almost universally accepted view, turbulent flows at high Reynolds numbers are more ordered than laminar ones at low Reynolds numbers. This can be explained considering that in the first case a large percentage of energy is concentrated in the collective modes of hydrodynamic motions (Ebeling, 1989; Klimontovich, 1996). This point is confirmed by the S-Theorem that, when applied to the transition from laminar to turbulent flow, exhibits a positive value of the entropy difference between the two states. Indeed, as it is clearly shown by Klimontovich (1996), we have:

$T\left[S_{\text {lam }}-S_{\text {turb }}\right]=\frac{m n}{2}\left\langle(\delta u)^{2}\right\rangle>0$.

where $\left\langle(\delta u)^{2}\right\rangle$ depends the sum of the diagonal elements in the Reynolds stress tensor. Thus, steady turbulent motions are more ordered than laminar ones, i.e. the transition to turbulence is an example of self-organization process emerging from a collective dynamics. However, the emergence of selforganization in turbulence flows can also be understood in terms of an increase in coherence in macroscopic motions accompanied by a decrease in the degree of spatial symmetries.

In the previous work on magnetic field intensity fluctuations by Consolini (2010) it was observed an increase in the degree of order with radial distance. This result was read in terms of an increasing relevance of magnetic coherent structures with radial distance. As noted in Bruno et al. (2006) there could be two different mechanisms responsible for the increasing relevance of coherent magnetic structures: (i) these could be a byproduct of Alfvénic turbulence or (ii) the remnants of structures produced at the Sun. Although Consolini (2010) suggested that coherent structures may be generated from turbulence evolution with radial distance, the hypothesis that coherent magnetic structures are remnants of structures produced at the solar surface cannot be excluded in light of our results on the decrease of the relative order in the turbulent fluctuation field. Indeed, the decaying/degradation of the solar wind turbulence with radial distance, which is accompanied by a decrease in the turbulent fluctuation amplitude, could imply the emergence of the underlining solar wind structures originated at the Sun. Clearly, at the present stage this aspect is still only speculative and deserves a more accurate analysis.

In conclusion, we clearly show that the evolution of slow solar wind turbulence with radial distance is accompanied by a degradation process. More work is necessary to extend these results to different solar wind conditions, although the extension to fast streams is not straightforward.

\section{Appendix A}

\section{On the meaning of the effective temperature $T_{\text {eff }}$}

To clarify the meaning of the effective temperature $T_{\text {eff }}$ as a measure of the degree of order, a simple illustrative example, that can also be found in Klimontovich (1991), is here extensively discussed

Let it be $f_{0}(v)$ and $f_{1}(v)$ the velocity distribution functions for two 1-D systems consisting of particles of mass $m$ and at the same temperature $T$, i.e.

$$
\begin{aligned}
& f_{0}(v)=\sqrt{\frac{m}{2 \pi \kappa_{\mathrm{B}} T}} \exp \left(-\frac{m v^{2}}{2 \kappa_{\mathrm{B}} T}\right), \\
& f_{1}(v)=\sqrt{\frac{m}{2 \pi \kappa_{\mathrm{B}} T}} \exp \left(-\frac{m(v-u)^{2}}{2 \kappa_{\mathrm{B}} T}\right),
\end{aligned}
$$

where the velocity $u$ is relative to a coherent bulk motion. Consider the distribution function $f_{0}$ as the reference one. According to Eq. (3), we introduce the effective Hamiltonian $H_{\text {eff }}$,

$H_{\mathrm{eff}}=-\ln f_{0} \simeq \frac{m v^{2}}{2 \kappa_{\mathrm{B}} T}$,

where the last equality is valid unless of a constant factor. From this expression we evaluate the mean effective energy for the two states;

$$
\begin{aligned}
\left\langle H_{\mathrm{eff}}\right\rangle_{0} & =\frac{1}{2}, \\
\left\langle H_{\mathrm{eff}}\right\rangle_{1} & =\frac{\kappa_{\mathrm{B}} T+m u^{2}}{2 \kappa_{\mathrm{B}} T} .
\end{aligned}
$$

These two states do not have the same mean effective energy, being $\left\langle H_{\text {eff }}\right\rangle_{1}>\left\langle H_{\text {eff }}\right\rangle_{0}$, and, consequently it is not possible to evaluate directly the relative entropy $\delta S$.

According to the Klimontovich's S-Theorem procedure, we can renormalize the distribution of the reference state, represented by $f_{0}$, so that the renormalized distribution $\tilde{f}_{0}$ acquires the same mean effective energy of the state " 1 ". To do this, following the renormalization procedure, we introduce an effective temperature $T_{\text {eff }}$ (see Eqs. 3 and 5) and write the new renormalized distribution $\tilde{f}_{0}$ in the form,

$$
\tilde{f}_{0}(v)=\sqrt{\frac{m}{2 \pi \kappa_{B} T_{\text {eff }}}} \exp \left(-\frac{m v^{2}}{2 \kappa_{\mathrm{B}} T_{\text {eff }}}\right), \quad T_{\text {eff }}=\tau T .
$$


Taking into account Eq. (6), we get

$\tau \kappa_{\mathrm{B}} T=\kappa_{\mathrm{B}} T+m u^{2} \rightarrow \tau>1$.

Thus, to have the same mean effective energy, the effective temperature $T_{\text {eff }}$ of the normalized reference state must be higher than that of the state " 1 ", $T_{\text {eff }}>T$. This means that while in the case of the reference state " 0 ", the energy is spent all in uncorrelated motions (thermal energy), for the state "1", part of the energy is spent in correlated motion (the mean kinetic drift energy). Consequently, the level of physical chaos in the state " 0 ", is higher than in the state " 1 ", and the energy spent in the collective motions, which is a signature of self-organization, yields a state of higher degree. This result is also confirmed by the computation of the entropy variation $\delta S$, evaluated using Eq. (8), which results

$\delta S=\tilde{S}_{0}-S_{1}=\frac{1}{2} \ln \tau$.

Hence, being $\tau>1$ it follows $\delta S>1$, i.e. the state " 1 ", is associated with an entropy reduction and with a higher degree of order. Equation (A8) can be also written in the form,

$\delta S=\frac{1}{2} \ln \left(1+\frac{m u^{2}}{\kappa_{\mathrm{B}} T}\right)$,

so that it is evident that the quantity $m u^{2} / \kappa_{\mathrm{B}} T$ acts as an order parameter, getting $\delta S=0$ in the limit $u \rightarrow 0$.

Acknowledgements. We are indebted with B. Bavassano (INAFIFSI, Italy) for useful discussions. G. Consolini thanks his colleague S. Savin for having brought to his attention the large work of Yu. L. Klimontovich on statistical physics of far-from-equilibrium open systems. We acknowledge the Ulysses PIs of FGM and SWOOPS (A. Balogh - Imperial College, London - and D. McComas - Southwest Res. Inst., USA), M. Lancaster and C. Tranquille of the Ulysses Data System (ESA-ESTEC, NL), the NASACDAweb service staff (USA), and J. H. King and N. Papitashvili of the NSSDC (USA) for the data (available on web) used in this work.

Guest Editor M. Balikhin thanks Z. Voros, M. Leubner, and another anonymous referee for their help in evaluating this paper.

\section{References}

Badii, R. and Politi, A.: Complexity: hierarchical structures and scaling in physics, Cambridge University Press, 1997.

Bavassano, B., Dobrowolny, M., Fanfoni, G., Mariani, F., and Ness, N. F.: Radial evolution of power specta of interplanetary Alfvnic turbulence, J. Geophys. Res., 87, 3617-3622, doi:10.1029/JA087iA05p03617, 1982a.

Bavassano, B., Dobrowolny, M., Mariani, F., and Ness, N. F.: Statistical properties of MHD fluctuations associated with high-speed streams from Helios 2 observations, Solar Phys., 78, 373-384, doi:10.1007/BF00151617, 1982b.

Bavassano, B., Pietropaolo, E., and Bruno, R.: Alfvénic turbulence in polar wind: A statisical study on cross-helicity and residual energy variations, J. Geophys. Res., 105, 12697-12704, doi:10.1029/2000JA900004, 2000.
Borovsky, J. E.: Flux tube texture of the solar wind: Strands of the magnetic carpet at 1 AU?, J. Geophys. Res., 113, A08110, doi:10.1029/2007JA012684, 2008.

Bruno, R. and Carbone, V.: The solar wind as a turbulence laboratory, Living Rev. Sol. Phys., 2, 4, 2005.

Bruno, R., Carbone, V., Veltri, P., Pietropaolo, E., and Bavassano, B.: Identifying intermittency events in the solar wind, Planet. Space Sci., 49, 1201-1210, doi:10.1016/S0032-0633(01)000617, 2001.

Bruno, R., Bavassano, B., D’Amicis, R., Carbone, V., SorrisoValvo, L., and Pietropaolo, E.: On the radial evolution of Alfvénic turbulence in the solar wind, Space Sci. Rev., 122, 321328, doi:10.1007/s11214-006-5232-8, 2006.

Consolini, G.: Relative degree of order in radial evolution of solar wind fluctuations, in: 12th International Solar Wind Conference, edited by: Maksimovic, M., Issautier, K., Meyer-Vernet, N., Moncuquet, M., and Pantellini, F., AIP Conf. Proc., 1216, 120-123, 2010.

Consolini, G., Bavassano, B., and De Michelis, P.: A probabilistic approach to heterogeneity in space plasmas: the case of magnetic field intensity in solar wind, Nonlin. Processes Geophys., 16, 265-273, doi:10.5194/npg-16-265-2009, 2009.

Ebeling, W.: On the entropy of dissipative and turbulent structures, Phys. Scripta, T25, 238-242, doi:10.1088/00318949/1989/T25/043, 1989.

Ebeling, W. and Klimontovich, Yu. L.: Self Organization and Turbulence in Liquids, Teubner, Leipzig, 1984.

Freeman, J. W., Totten, T., and Ayra, S.: A determination of polytropic index of the free streaming solar wind using improved temperature and density radial power-law indices, Eos Trans. AGU, 73, 238, 1992.

Goldstein, B. E., Neugebauer, M., Phillips, J. L., Bame, S., Gosling, J. T., McComas, D., Wang, Y.-M., Sheeley, N. R., and Suess, S. T.: ULYSSES plasma parameters: latitudinal, radial, and temporal variations, Astron. \& Astrophys., 316, 296-303, 1996.

Horbury, T. S., Balogh, A., Forsyth, R. J., and Smith, E. J.: Anisotropy of inertial range turbulence in the polar heliosphere, Geophys. Res. Lett., 22, 3405-3408, doi:10.1029/95GL03012, 1995.

Klimontovich, Yu. L.: Entropy decrease in process of self organization. S-Theorem, Pis'ma v ZhTP, 9, 1089-1093, 1983.

Klimontovich, Yu. L.: Entropy and entropy production in the laminar and the turbulent flows, Pis'ma v ZhTP, 10, 80-83, 1984.

Klimontovich, Yu. L.: Turbulent Motion and the Sructure of Chaos. A new approach to the statistical theory of open systems, Kluwer Academic Publishers, 1991.

Klimontovich, Yu. L.: Statistical Theory of Open Systems, Vol.1, Kluwer Academic Publishers, 1995.

Klimontovich, Yu. L.: Is turbulent motion chaos or order? Is the hydrodynamic or kinetic description of turbulent motion more natural?, Physica B, 228, 51-62, doi:10.1016/S0921-4526(96)003389, 1996.

Mariani, F., Bavassano, B., Villante, U., and Ness, N. F.: Variations of the Occurrence Rate of Discontinuities in the Interplanetary Magnetic Field, J. Geophys. Res., 78, 8011-8022, doi:10.1029/JA078i034p08011, 1973.

Marino R., Sorriso-Valvo, L., Carbone, V., Noullez, A., Bruno, R., and Bavassano, B.: Heating the solar wind by a magnetohydrodynamic turbulent energy cascade, Astrophys. J., 677, L71-L74, 
doi:10.1086/587957, 2008.

McCracken, K. G. and Ness, N. F.: The Collimation of Cosmic Rays by the Interplanetary Magnetic Field, J. Geophys. Res., 71, 3315-3318, doi:10.1029/JZ071i013p03315, 1966.

Ness, N. F., Scearce, C. S., and Cantarano, S.: Preliminary Results from the Pioneer 6 Magnetic Field Experiment, J. Geophys. Res., 71, 3305-3313, doi:10.1029/JZ071i013p03305, 1966.

Nicolis, G. and Nicolis, C.: Foundations of Complex Systems. Nonlinear Dynamics, Statistical Physics, Information and Prediction, World Scientific Publishing Co. Pte. Ltd., 2007.

Prigogine, I. and Stengers, I.: Order out of chaos, Heinemamm, London, 1984.

Roberts, D. A., Goldstein, M. L., Klein, L. W., and Matthaeus, W. H.: Origin and evolution of fluctuations in the solar wind: Helios observations and Helios-Voyager comparisons, J. Geophys. Res., 92, 12023-12035, doi:10.1029/JA092iA11p12023, 1987.

Russell, C. T.: Solar wind and interplanetary magnetic field: A tutorial, Space Weather, Geophysical Monograph, 125, 2001.

Schwenn, R.: The average solar wind in the inner heliosphere: Structure and slow variations, in: Solar Wind FIVE, NASA Conf. Publ., CP-2280, 485, 1983.
Tu, C.-Y. and Marsch, E.: Evidence for a Background Spectrum of Solar Wind Turbulence in the Inner Heliosphere, J. Geophys. Res., 95, 4337-4341, doi:10.1029/JA095iA04p04337, 1990.

Tu C.-Y. and Marsch, E.: A Model of Solar Wind Fluctuations with Two Components: Alfvén Waves and Convective Structures, J. Geophys. Res., 98, 1257-1276, doi:10.1029/92JA01947, 1993.

Tu, C.-Y., Pu, Z.-Y., and Wei, F.-S.: The power spectrum of interplanetary Alfvénic fluctuations: deviation of the governing equation and its solution, J. Geophys. Res., 89, 9695-9702, doi:10.1029/JA089iA11p09695, 1984.

Tu, C.-Y., Marsch, E., and Thieme, K. M.: Basic properties of solar wind MHD turbulence near $0.3 \mathrm{AU}$ analysed by mean of Elsässer variables, J. Geophys. Res., 94, 11739-11759, doi:10.1029/JA094iA09p11739, 1989.

Verma, M. K., Roberts, D. A., and Goldstein, M. L.: Turbulent heating and temperature evolution in the solar wind plasma, J. Geophys. Res., 100, 19839-19850, doi:10.1029/95JA01216, 1995. 